\title{
Comparação entre o HOP TEST e outros testes utilizados na alta de pacientes com lesão de ligamento cruzado anterior LCA)
}

\author{
Comparison between the Hop Test and other tests used in the \\ release of patients with injury of Anterior Cruciate Ligament (ACL)
}

\author{
Demétryus Corrêa de Farias Neto' \\ Kaio Primo Manso ${ }^{2}$ \\ Keila de Nazaré Madureira Batista ${ }^{3}$
}

\section{RESUMO}

A lesão de Ligamento Cruzado Anterior (LCA) é uma das lesões ligamentares mais comuns na prática desportiva, principalmente no futebol, devido à mecânica do esporte que acaba favorecendo casos de entorse, $\mathrm{e}$ também através de trauma, onde o contato é intenso entre os participantes. Ao fim do período de reabilitação o lesionado de LCA é submetido a exames para avaliar se está apto para o retorno à prática esportiva, dentre eles o principal é o Dinamômetro Isocinético, porém, devido seu alto custo, muitas vezes acabam por utilizar outros testes mais viáveis para a realidade de muitos locais de reabilitação, como o hop test. Objetivo: analisar na literatura a utilização do hop test como um dos métodos utilizados para alta de lesionados de Ligamento Cruzado Anterior, sua incidência e eficácia como meio avaliativo, frente aos demais métodos utilizados devido sua baixa demanda de tempo e recursos para a aplicação. Métodos: revisão na literatura em bases gratuitas sobre o hop test sendo utilizado em lesionados de Ligamento Cruzado Anterior (LCA) comparando com outros testes utilizados neste grupo, nos idiomas português e inglês. Resultados: foram encontrados somente 3 estudos abrangendo os requisitos do estudo. Conclusão: o hop test se mostra mais confiável comparado a uma análise subjetiva, e bom comparado ao dinamômetro isocinético, porém se faz necessário a realização de mais estudos sobre o tema.

\section{PALAVRAS-CHAVE}

Hop test; Isocinético; Esportes; Lesão; Ligamento Cruzado Anterior.

\footnotetext{
${ }^{1}$ Discente do Curso de Fisioterapia na Universidade Federal do Pará.

2 Discente do Curso de Fisioterapia na Universidade Federal do Pará.

${ }^{3}$ Universidade Federal do Pará - UFPA.
} 


\section{ABSTRACT}

Introduction: the injury of Anterior Cruciate Ligament ( $A C L)$ is one of the most common ligament injuries in sports, particularly in football due to the mechanics of the sport that ends up favoring cases of sprains, and also through trauma, where contact is intense among participants. At the end of the rehabilitation period, who suffered ACL injuries is undergoing tests to determine if it is fit to return to sports, among them the main thing is the Isokinetic Dynamometer, however, due to its high cost, often end up using other more viable test for the reality of many rehabilitation sites such as the hop test. Objective: To analyze in the literature the use of the hop test as one of the methods used in the release of patients with injury of $A C L$, their impact and effectiveness as a means evaluation, compared to other methods used due to its low demand of time and resources for the application. Methods: review of the literature on free bases on the hop test being used in the injury of Anterior Cruciate Ligament $(\mathrm{ACL})$ compared to other tests used in this group, in languages portuguese and inglish. Results: we found only three studies covering the requirements of the study. Conclusion: the hop test proves more reliable compared to a subjective analysis, and good compared to isokinetic dynamometer, but it is necessary to carry out more studies on the subject.

\section{KEYWORDS}

Hop test, Isokinetic, Sports, Injury, Anterior Cruciate Ligament. 


\section{INTRODUÇÃO}

O joelho é uma articulação móvel que sustenta peso e sua estabilidade depende quase que inteiramente de seus ligamentos, meniscos e músculos associados. É uma articulação submetida a grandes esforços, pois se localiza entre dois braços de alavanca, o fêmur e a tíbia, e é essencial para as atividades diárias como ficar de pé, caminhar, subir e descer escadas. Também é uma articulação principal para esportes que envolvem corrida, salto, chute e mudança de direção. Neste contexto, a articulação do joelho precisa ser móvel; entretanto esta mobilidade a torna suscetível a lesões durante essas atividades na prática esportiva. ${ }^{1,2}$

Dentre os ligamentos do joelho que mais sofrem lesões no desporto, o LCA se destaca, principalmente na prática do futebol, devido à mecânica do esporte que acaba favorecendo casos de entorse, e também através de trauma. ${ }^{3}$

Independente do grau da lesão, o fisioterapeuta irá trabalhar no controle da dor e redução de edema, correção da biomecânica, estabilidade de movimento através de treinamento de força e equilíbrio, propriocepção, força muscular, além de exercícios que ajudam na prevenção de possíveis lesões. ${ }^{2,3}$

Na prática esportiva, o período de reabilitação dos atletas acaba sendo exigido no mínimo de tempo possível, o que pode acabar precipitando a decisão de alta, podendo ocasionar nova lesão. Os protocolos de reabilitação após cirurgia em caso de ruptura total do LCA costumam durar de três a seis meses, com retorno às práticas esportivas podendo ocorrer de seis a oito meses. ${ }^{3}$

Na prática clinica da fisioterapia percebe-se que não há um acordo entre os fisioterapeutas quanto aos protocolos de alta e métodos de avaliação para pacientes lesados de LCA, muito se vê a utilização de treinamentos funcionais como parâmetros para alta do paciente, sendo que tudo se torna muito subjetivo. Ao realizar pesquisas em revistas cientificas podemos constatar que há um déficit de publicações cientifica quando se trata de protocolos de alta para lesados de LCA, mesmo esta sendo uma lesão comum.

Dentre os meios de se ter uma conclusão de segurança no joelho há o hop test, que é um teste para avaliação da força e da confiança nos membros inferiores (MMII), comparando o membro lesado com o não lesado, que pode ser utilizado na clínica com pequeno gasto de tempo e mínima demanda financeira. $\mathrm{O}$ hop test e suas variações têm sido amplamente utilizadas para avaliar o retorno ao nível funcional do joelho lesado, principalmente pós-reconstrução de ligamento cruzado anterior (LCA). ${ }^{4}$

O presente estudo tem por objetivos analisar na literatura a utilização do hop test como um dos métodos utilizados para alta de lesionados de Ligamento Cruzado Anterior, sua incidência e eficácia como meio avaliativo, frente aos demais métodos utilizados devido sua baixa demanda de tempo e recursos para a aplicação.

\section{MATERIAL E MÉTODO}

Trata-se de um estudo de revisão, foi realizado com base em pesquisas efetuadas nas principais revistas gratuitas (Scielo, Lilacs, Pubmed*) utilizando os termos: Hop test, LCA, Ligamento Cruzado Anterior, Anterior Cruciate Ligament, ACL, Protocolos de Alta, Joelho, Lesão. Com base nos artigos encontrados foram selecionados aqueles que apresentavam: lesões durante a prática esportiva, somente lesão de LCA sem associação com lesão meniscal, cirurgias sem complicações, protocolos de tratamento que levaram de 6 a 8 meses, estudos com dinamômetro isocinético, utilização do hop test na lesão de LCA e em comparação com o dinamômetro isocinético, e como fator primário ser a partir de 2005.

\section{RESULTADOS}

Neste estudo de revisão foram coletados 17 artigos abrangendo os requisitos, destes apenas 3 ficaram de acordo com os critérios de inclusão necessários, comparando o dinamômetro isocinético com o hop test, ultizando o hop test como avaliação do joelho após lesão de LCA, e a partir de 2005. 
Tabela 1- Caracterização dos estudos

\begin{tabular}{|c|c|c|c|c|c|}
\hline Autor/Ano & Amostragem & $\begin{array}{l}\text { Tipo de } \\
\text { estudo }\end{array}$ & Intervenção & Avaliação & Conclusão \\
\hline $\begin{array}{l}\mathrm{D}^{\prime} \text { Alessandro } \\
\mathrm{RL} \text { et al, } 2005^{4}\end{array}$ & $\begin{array}{l}\mathrm{n}=30 \\
\begin{array}{l}\text { Atletas de vôlei } \\
\text { sem lesão }\end{array} \\
\text { homens }=22 \\
\text { mulheres }=8\end{array}$ & $\begin{array}{l}\text { Estudo } \\
\text { compara- } \\
\text { tivo }\end{array}$ & $\begin{array}{l}\text { Todos foram sub- } \\
\text { metidos ao dina- } \\
\text { mômetro isocinéti- } \\
\text { co (velocidades de } \\
60 \% \text { e } 300 \% \text { s) e ao } \\
\text { hop test em ambos } \\
\text { os MMII. }\end{array}$ & $\begin{array}{l}\text { Hop Test: Os atletas fo- } \\
\text { ram informados sobre } \\
\text { o procedimento do sal- } \\
\text { to e solicitados a saltar } \\
\text { a maior distância possí- } \\
\text { vel com cada membro } \\
\text { inferior.Os saltos foram } \\
\text { executados por três ve- } \\
\text { zes com cada MI. O } \\
\text { procedimento foi en- } \\
\text { tão reproduzido para o } \\
\text { Ml esquerdo. O melhor } \\
\text { salto com cada mem- } \\
\text { bro foi utilizado para } \\
\text { fins estatísticos. } \\
\text { Dinamômetro isocinéti- } \\
\text { co: foi realizada por } \\
\text { um dinamômetro isoci- } \\
\text { nético Biodex System } \\
3-\text { Pro (Nova lorque, } \\
\text { EUA). Antes do teste os } \\
\text { atletas realizavam um } \\
\text { aquecimento em bici- } \\
\text { cleta ergométrica por } \\
10 \text { minutos, seguido de } \\
\text { quatro séries de } 20 \text { se- } \\
\text { gundos de exercícios de } \\
\text { alongamento para os } \\
\text { músculos isquiotibiais e } \\
\text { quadríceps femoral. A } \\
\text { amplitude de teste foi } \\
\text { limitada em } 100^{\circ} \text {, com } \\
\text { início em } 110^{\circ} \text { de fle- } \\
\text { xão e término em } 10^{\circ} \\
\text { de flexão de joelho. } \\
\text { Os testes foram realiza- } \\
\text { dos no modo concên- } \\
\text { trico sendo cinco repe- } \\
\text { tições para } 60^{\circ} / s \text { e cin- } \\
\text { co repetições para } \\
300^{\circ} \text { s. Entre cada ve- } \\
\text { locidade era dado um } \\
\text { intervalo de } 30 \text { segun- } \\
\text { dos de repouso. }\end{array}$ & $\begin{array}{l}\text { Foi observada somente } \\
\text { uma correlação baixa do } \\
\text { dinamômetro isocinético } \\
\text { com o hop test entre défi- } \\
\text { cit de pico de torque e de } \\
\text { trabalho a } 600 / s \text { com o } \\
\text { déficit da distância salta- } \\
\text { da entre MMII. Foi obser- } \\
\text { vada baixa associação en- } \\
\text { tre a performance muscu- } \\
\text { lar e a distância saltada } \\
\text { no hop test exceto no } \\
\text { membro inferior direito } \\
\text { de mulheres. }\end{array}$ \\
\hline $\begin{array}{l}\text { Logerstedt et } \\
\text { al, } 2012^{5}\end{array}$ & $\begin{array}{l}\mathrm{n}=120 \\
\text { Com idades en- } \\
\text { tre } 15-54 \text { anos. }\end{array}$ & $\begin{array}{l}\text { Estudo de } \\
\text { Coorte }\end{array}$ & $\begin{array}{l}\text { Pacientes foram } \\
\text { submetidos a } 4 \text { hop } \\
\text { e a questionários de } \\
\text { auto relato no perí- } \\
\text { odo pré-operatório } \\
\text { e } 6 \text { meses após a } \\
\text { cirurgia. Foram } \\
\text { acompanhados } 1 \\
\text { ano após a cirurgia } \\
\text { e novamente apli- } \\
\text { cado o questioná- } \\
\text { rio. }\end{array}$ & $\begin{array}{l}\text { Foram realizados } 4 \text { tes- } \\
\text { tes unipodais: o hop } \\
\text { único para a distância } \\
\text { (single-hop), hop cruza- } \\
\text { do para a distância } \\
\text { (crossover hop), hop tri- } \\
\text { plo para a distância (tri- } \\
\text { plo hop), e o hop de } 6 \\
\text { metros cronometrados } \\
\text { (6-m timed hop) }\end{array}$ & $\begin{array}{l}\text { Os pacientes que foram } \\
\text { classificados como tendo a } \\
\text { função do joelho auto-re- } \\
\text { portados dentro da nor- } \\
\text { malidade no } 1^{\circ} \text { ano de } \\
\text { acompanhamento teve ín- } \\
\text { dices significativamente } \\
\text { maiores no hop test cruza- } \\
\text { do e no de } 6 \text { m cronome- } \\
\text { trado } 6 \text { meses após a cirur- } \\
\text { gia. Prevendo significativa- } \\
\text { mente a função do joelho } \\
\text { um ano após a reconstru- } \\
\text { ção do LCA. }\end{array}$ \\
\hline
\end{tabular}




\begin{tabular}{|c|c|c|c|c|c|}
\hline Autor/Ano & Amostragem & \begin{tabular}{|l|} 
Tipo de \\
estudo
\end{tabular} & Intervenção & Avaliação & Conclusão \\
\hline $\begin{array}{l}\text { Logerstedt et } \\
\text { al, } 2013^{6}\end{array}$ & $\begin{array}{l}\mathrm{n}=102 \\
\text { Com idades de } \\
15-53 \text { anos, } \\
\text { praticantes re- } \\
\text { gulares de ati- } \\
\text { vidade física }\end{array}$ & $\begin{array}{l}\text { Estudo de } \\
\text { Coorte }\end{array}$ & $\begin{array}{l}\text { Os indivíduos fo- } \\
\text { ram submetidos a } \\
\text { testes de força do } \\
\text { quadríceps, hop } \\
\text { test e questioná- } \\
\text { rios de auto relato. }\end{array}$ & $\begin{array}{l}\text { Teste de força do qua- } \\
\text { dríceps: consistiu de } \\
\text { contração isométrica } \\
\text { voluntária máxima (Cl- } \\
\text { VM) do quadríceps } \\
\text { com a técnica de so- } \\
\text { breposição explosão. } \\
\\
\text { Teste de unipodal: Hop } \\
\text { test- o hop único para a } \\
\text { distância (single-hop), } \\
\text { hop cruzado para a dis- } \\
\text { tância (crossover hop), } \\
\text { hop triplo para a dis- } \\
\text { tância (triplo hop), e o } \\
\text { hop de } 6 \text { metros crono- } \\
\text { metrados (6-m timed } \\
\text { hop) } \\
\text { Questionários de au- } \\
\text { to-relato: pacientes } \\
\text { completaram três } \\
\text { questionários de au- } \\
\text { to-relato: Knee Out- } \\
\text { come Survey-Activities } \\
\text { of Daily Living Scale } \\
\text { (KOS-ADLS), the Inter- } \\
\text { national Knee Docu- } \\
\text { mentation Committee } \\
\text { 2000 Subjective Knee } \\
\text { Form (IKDC2000), and } \\
\text { the Global Rating Scale } \\
\text { of Perceived Function } \\
\text { (GRS) }\end{array}$ & $\begin{array}{l}\text { Foi constatado que o qua- } \\
\text { dríceps lesionado melho- } \\
\text { rou seu nível de força } \\
\text { mais que o não lesado. } \\
\text { No hop test simples, triplo } \\
\text { e no de } 6 \text { metros, o mem- } \\
\text { bro lesionado obteve me- } \\
\text { Ihora em relação ao não } \\
\text { lesado se comparados a } \\
\text { avaliação inicial. }\end{array}$ \\
\hline
\end{tabular}

\section{DISCUSSÃo}

O dinamômetro isocinético possui boa validade e confiabilidade, sendo frequentemente utilizado no estudo da função muscular dinâmica, principalmente na avaliação pós-lesão. No entanto, sua utilização na prática clínica se torna inviável devido seu alto custo, além de sua interpretação de resultados da avaliação requerer experiência do avaliador e demanda de tempo para a execução do teste e análise de dados. Com isso, o hop test e suas variações têm sido amplamente utilizados para avaliar o retorno ao nível funcional do joelho lesado, principalmente pós -reconstrução de ligamento cruzado anterior (LCA), pois mesmo não permitindo uma análise detalhada da função do MMII, como no dinamômetro isocinético, ele permite uma triagem geral durante a avalição do MMII lesado na prática clínica com pequeno gasto de tempo e mínima demanda financeira. ${ }^{4}$

D'Alessandro et al (2005) ${ }^{4}$ se propôs a verificar se existe associação entre o hop test e dados da função muscular fornecidos pela avaliação do joelho do dinamômetro isocinético em atletas profissionais. Seu estudo verificou uma associação baixa, mas significativa, apenas na velocidade de $60 \% \mathrm{~s}$, tanto para o torque máximo com distância saltada quanto para trabalho com distância saltada. Nesta velocidade bai$\mathrm{xa}$, consegue-se maior geração de torque, aproximando-se mais do desempenho muscular máximo dos atletas, assim, sua significância neste quesito poderá ser um indicador de utilização para a alta e retorno gradual as atividades esportivas com seu resultado de confiança no membro lesionado, desde que se realizem outros estudos que confirmem este resultado devido ao revés deste ter sido realizado com uma população sem lesão, necessitando de estudos com amostragem maior, atletas e não atletas, e com lesão de LCA para se confirmar com precisão sua associação.

No entanto, algumas especificações dos métodos em questão deste estudo dificultam uma análise precisa, visto que o dinamômetro isocinético foi realiza- 
do apenas no modo concêntrico e em cadeia cinética aberta, enquanto que o hop test permite uma ação muscular excêntrica que antecede o salto podendo potencializá-lo, e é realizado em cadeia cinética fechada favorecendo a utilização de métodos compensatórios, podendo interferir no resultado da associação real entre ambos os métodos.

Logerstedt et al (2012) $)^{5}$ traz o uso do hop test como preditor para a recuperação da lesão de LCA, em seu estudo é constatado que os pacientes que obtiveram um score elevado no hop test 6 meses após a cirurgia apresentaram um melhor prognostico um ano depois, relatando que o membro lesionado estava totalmente dentro da normalidade. Sendo assim testes de salto no período pré-operatório não são preditivos de função do joelho após reconstrução do LCA. Testes de salto são clinicamente úteis para prever o futuro 6 meses após a reconstrução do LCA, pode-se prever quem vai ter a função do joelho dentro ou abaixo da faixa de normalidade no prazo médio de 1 ano após a reconstrução do LCA. Estes testes têm implicações consideráveis na tomada de decisão clínica em função do futuro do joelho, podem ser usados para informar o médico e paciente sobre provável prognóstico do paciente e no desenvolvimento da reabilitação direcionada para resolver as assimetrias dos membros e para promover a função normal do joelho.

Em estudo posterior Logerstedt et al $(2013)^{6}$ traz novamente a utilização do hop test como avaliação no período de 6 -12 meses após reconstrução do ligamento cruzado anterior, mas segundo os dados colhidos não apresentou a mesma taxa de mudança significativa.

\section{CONCLUSÃO}

Concluímos que o hop test se mostra mais confiável comparado a uma análise subjetiva, e bom com- parado ao dinamômetro isocinético. Porém, há a necessidade de mais estudos focando nos efeitos comparativos entre o hop test e demais métodos utilizados, sobretudo no uso de alta de lesão do ligamento cruzado anterior, pois a literatura não é clara quanto eficácia do uso do hop test na alta na ausência do dinamômetro isocinético, ou quanto a significância de comparação entre os métodos, sendo utilizado geralmente em pacientes saudáveis, além da elaboração de critérios mais semelhantes na elaboração dos estudos para a obtenção de resultados satisfatório.

\section{REFERÊNCIAS}

1 - RIBAS APG; LIMA MCAM. Avaliação Funcional em Pacientes Submetidos a Meniscectomia Artroscópica. Revista Ágora. Vol.16, n.2, Número Especial: I Seminário Integrado de Pesquisa e Extensão Universitária.

2 - TOOKUNI KS; BOLLIGER NETO R, et al. Análise comparativa do controle postural de indivíduos com e sem lesão do ligamento cruzado anterior do joelho. Acta Ortop Bras. Vol.13, n.3. 2005.

3 - BRIRO J; SOARES J; REBELO AN. Prevenção de lesões do ligamento cruzado anterior em futebolistas: revisão. Rev. bras. med. esporte; Vol.15, n.1, pp.62-69, jan.-fev, 2009.

4 - D'ALESSANDRO RL; SILVEIRA EAP, et al. Análise da associação entre a dinamometria isocinética da articulação do joelho e o salto horizontal unipodal, hop test, em atletas de voleibol. Rev Bras Med Esporte. Vol.11, n.5, pp. 271-275, 2005.

5 - LOGERSTEDT D; GRINDEM H; LYNCH A; EITZEN I; ENGEBRETSEN L; RISBERG MA. Single-legged hop tests as predictors of self-reported knee function after anterior cruciate ligament reconstruction: the Delaware-Oslo $\mathrm{ACL}$ cohort study. Am J Sports Med. Vol.40, n. 10, pp. 2348-2356, 2012.

6 - LOGERSTEDT D; LYNCH A; AXE MJ; SNYDER-MACKLER L. Symmetry restoration and functional recovery before and after anterior cruciate ligament reconstruction. Knee Surg Sports Traumatol Arthrosc. Vol.21, n.4, pp. 859-868, 2013. 